\title{
Is the dynamics of open quantum systems always linear?
}

\author{
Karen M. Fonseca Romero* Peter Talkner, and Peter Hänggi \\ Institut für Physik, Universität Augsburg, Universitätsstr.1, D 86315 Augsburg, Germany
}

(Dated: October 31, 2018)

\begin{abstract}
We study the influence of the preparation of an open quantum system on its reduced time evolution. In contrast to the frequently considered case of an initial preparation where the total density matrix factorizes into a product of a system density matrix and a bath density matrix the time evolution generally is no longer governed by a linear map nor is this map affine. Put differently, the evolution is truly nonlinear and cannot be cast into the form of a linear map plus a term that is independent of the initial density matrix of the open quantum system. As a consequence, the inhomogeneity that emerges in formally exact generalized master equations is in fact a nonlinear term that vanishes for a factorizing initial state. The general results are elucidated with the example of two interacting spins prepared at thermal equilibrium with one spin subjected to an external field. The second spin represents the environment. The field allows the preparation of mixed density matrices of the first spin that can be represented as a convex combination of two limiting pure states, i.e. the preparable reduced density matrices make up a convex set. Moreover, the map from these reduced density matrices onto the corresponding density matrices of the total system is affine only for vanishing coupling between the spins. In general, the set of the accessible total density matrices is nonconvex.

PACS numbers: 03.65.Yz, 05.30.Ch, 02.50.-r
\end{abstract}

\section{INTRODUCTION}

Closed systems are known to be an idealization. In general, real systems interact with their environment and exhibit properties that cannot be observed in finite closed systems, such as irreversibility of the time evolution and, related, the relaxation of observables toward stationary values and dephasing, or decoherence. Various techniques have been developed to treat the dynamics of open systems without explicitly considering the full Hamiltonian dynamics [1]. For example, effective equations for the reduced density matrix of the considered open system, known as master equations, have been proposed long ago 2, 3, 4] and still are of considerable interest because of their conceptional simplicity and potential usefulness [5, 6]. New challenges in this field of fundamental physics have come from nanotechnology 7] and quantum computing [8].

Any equation determining the time evolution of a density matrix has to obey several general properties which guarantee that the density matrix stays selfadjoint, positive and normalized in the course of time. These general properties still leave much freedom and, in order to further restrict possible dynamical laws, additional requirements for the dynamics have been postulated [9]. One seemingly natural property that often is assumed without even being mentioned is the linearity of the time evolution, which generally is understood as a consequence of the linearity of the Schrödinger and the Liouville-von Neumann equation for closed systems. This argument, however, only works by analogy and no proof of the necessity of this requirement is available. Just on the contrary Pechukas [10, 11] has recently shown that linearity may only hold if the initial state of the total system factorizes into a product of a density matrix for the open system and another one for the environment, and if a sufficient number of pure states can be prepared. The assumption of linearity is a prerequisite of a Markovian dynamics and of complete positivity [12, 13. These properties then lead to the mathematically well characterized class of Lindblad master equations. From the physical point of view, however, these equations suffer from certain deficiencies [10]. They are restricted to the regime of weak coupling between the considered system and its environment. In particular the weak coupling assumption will fail at sufficiently low temperatures $[14,15]$. Moreover, there are various general statistical mechanical properties that are incompatible with the assumption of a Markovian dynamics [16].

A few microscopic models of systems interacting with their environment can be reduced exactly to Lindblad master equations with time dependent coefficients [17, 18], thereby describing the single time non-Markovian reduced dynamics.

The dynamics of an open system is determined by both, the full dynamics of the considered system interacting with its environment and the initial state of the complete system. The significance of the initial state was emphasized

*On leave from Departamento de Física, Universidad Nacional, Bogotá, Colombia. 
in several works $[19,20,21,22,[23,24,25,26,27]$. In an experiment this initial state is imposed by a preparation procedure. Here we will only be concerned with equilibrium preparations that lead to a thermal equilibrium of the total system in the presence of external fields that only act on the system and that are switched off finally. In this way, the initial state is described by a canonical density matrix:

$$
\rho^{\mathbf{F}}=Z^{-1} \exp \left\{-\beta\left(H-\sum_{j} F_{j} X_{j}\right)\right\},
$$

where $\beta$ is the inverse temperature, $H$ the Hamiltonian governing the dynamics of the total system, $\mathbf{F}=\left(F_{j}\right)$ are external, i.e. classical, fields, $X_{j}$ the corresponding conjugate operators of the open system and $Z=$ $\operatorname{Tr} \exp \left\{-\beta\left(H+\sum_{j} F_{j} X_{j}\right)\right\}$ is the partition function of the total system. Here, Tr denotes the trace over the total system. In this way, initial states of the total system are reproducibly prepared. They correspond to a thermal equilibrium of the environment at a given temperature and to a state of the system that depends on the strengths of the external fields $F_{j}$. The set of density matrices that can be obtained upon variation of the fields forms the equilibrium preparation class. The reduced states belonging to this preparation class are determined by the trace over the environment, which is denoted by $\operatorname{Tr}_{B}$ :

$$
\rho_{S}^{\mathbf{F}}=\operatorname{Tr}_{B} \rho^{\mathbf{F}}
$$

The calculation of this trace is nontrivial in most cases and in general does not lead to the canonical distribution of the uncoupled system, $Z_{S}^{-1} \exp \left\{-\beta H_{S}\right\}[14,28,29,30]$ where $H_{S}$ is the Hamiltonian of the system in presence of the external fields and $Z_{S}$ the respective partition function. This particular form is only obtained in the limit of weak coupling between the system and the environment [3, 29, 31]. In the weak coupling limit, the equilibrium density matrix of the total system factorizes into a product of a system and an environment density matrix. This is an example of the factorizing preparation which leads to a product of a particular density matrix of the environment $\rho_{B}$ and an arbitrary density matrix $\rho_{S}$ of the system:

$$
\rho^{\mathrm{fac}}=\rho_{S} \rho_{B}
$$

The factorizing preparation is assumed in most theoretical investigations though it is often difficult, if not impossible, to realize it experimentally.

A more general class of preparations has been suggested in the context of the path integral approach to open systems [22]:

$$
\rho^{O}=\sum_{j} O_{j} \rho^{\mathbf{F}} O_{j}^{\prime}
$$

where $\rho^{\mathbf{F}}$ is defined as in the equilibrium preparation, eq. (1), and $O_{j}$ and $O_{j}^{\prime}$ are operators that only act on the system's Hilbert space. For applications of this preparation class we refer the reader to Ref. [22].

The state of the open system after the preparation results as the trace over the environment of the density matrix of the full system at that time, i.e.

$$
\rho_{S}(t)=\operatorname{Tr}_{B} U(t) \rho U^{\dagger}(t)
$$

where

$$
U(t)=\exp \left\{-\frac{i}{\hbar} H t\right\}
$$

is the unitary time evolution operator of the full system and $\rho(0)=\rho$ the density matrix resulting from the preparation.

Requiring an affine time evolution of the reduced density matrix $\rho_{S}(t)$ means that $\rho_{S}(t)$ is the sum of a term linear in, and one independent of $\rho_{S}$, i.e.

$$
\rho_{S}(t)=T(t) \rho_{S}+I(t)
$$

where $T(t)$ is a linear operator and $\mathrm{I}(\mathrm{t})$ is independent of $\rho_{S}$. According to eq. (5) the reduced density matrix at time $t$ is a linear image of the initial full density matrix $\rho$ under the successive action of the unitary time evolution of the full system and the operation of the trace. In order to obtain a map from the initial reduced density matrix $\rho_{S}$ to its 
value at a later time $t$ we introduce the blow-up map $R\left(\rho_{S}\right)$ that assigns to each reduced initial density matrix $\rho_{S}$ one belonging to the total system:

$$
\rho=R\left(\rho_{S}\right)
$$

Its particular form depends on the initial preparation of the system. Expressing the initial total density matrix $\rho$ with the help of the blow-up map by the reduced initial state one obtains the reduced time evolution of the system:

$$
\rho_{S}(t)=\operatorname{Tr}_{B} U(t) R\left(\rho_{S}\right) U^{\dagger}(t) .
$$

An affine time evolution as described in eq. (77) will result only from an affine blow-up map. So one may ask under which conditions the blow-up map is affine.

Because a density matrix is a positive normalized operator, we require that the blow-up map $R$ acts on a convex set of reduced density matrices, i.e. a set that contains with each pair $\rho_{S 1}$ and $\rho_{S 2}$ all convex combinations $\lambda \rho_{S 1}+(1-\lambda) \rho_{S 2}$ for all $0<\lambda<1$. We assume next that the considered preparation provides such a convex set of reduced density matrices. For any particular preparation one has to check this property. We then further may ask under which conditions $R$ preserves the convexity condition, put differently under which conditions a full density matrix that corresponds to a convex combination of reduced density matrices again is given by a convex combination. Then the preparation class also forms a convex set. For such a convex blow-up map one obtains:

$$
R\left(\lambda \rho_{S, 1}+(1-\lambda) \rho_{S, 2}\right)=\lambda R\left(\rho_{S, 1}\right)+(1-\lambda) R\left(\rho_{S, 2}\right) .
$$

This then implies that the blow-up map is affine:

$$
R\left(\rho_{S}\right)=L \rho_{S}+\chi
$$

where $L$ is a linear operator that maps reduced density matrices onto full density matrices and $\chi$ is an operator of the full system. A proof of this statement is given in the Appendix A

We note that the mixing parameter $\lambda$ has to be identical on the left and on the right hand side of the eq. (10). This is a consequence of the fact that the trace of $R\left(\rho_{S}\right)$ over the Hilbert space of the environment must coincide with the system density matrix $\rho_{S}$ :

$$
\operatorname{Tr}_{B} R\left(\rho_{S}\right)=\rho_{S}
$$

For the factorizing preparation (3) the blow-up map $R$ is always linear. It simply acts as the multiplication by the reference environment density operator $\rho_{B}$. In the case of a classical system dynamics the role of density matrices is taken over by probability densities defined on the respective phase space. Then, any preparation can be characterized by a conditional probability density $\rho\left(\mathbf{x} \mid \mathbf{x}_{S}\right)$ for the state $\mathbf{x}$ of the total system given the state $\mathbf{x}_{S}$ of the system [19, 20, 21]. The corresponding blow-up map $R$ is then given by the multiplication with this conditional probability and, hence, is always linear. No such simple construction scheme is available in quantum mechanics and Pechukas 10 ] showed that the factorizing preparation is the only one for which the blow-up map is linear, provided that a sufficient number of pure states of the reduced system are contained in the preparation class. For the convenience of the reader the precise formulation of the theorem and a proof is given in the Appendix B

In the present work, we will consider the influence of the preparation of an open quantum system on its reduced time evolution by means of the simple example of two interacting spins. One of those is considered as the system, the other one plays the role of the environment. The second spin is only a very crude caricature of a true environment which clearly fails to cause dissipation or dephasing in the system because of its finiteness. Nevertheless, it suffices to illustrate the influence of the preparation on the subsequent dynamics of the system.

We assume that the total system starts from an equilibrium preparation, i.e. that its initial state is described by a density matrix of the form of eq. (11). It will be shown that in general this preparation renders the time evolution of the reduced system nonlinear.

\section{TWO SPINS}

Both interacting spins $\boldsymbol{\sigma}_{1}=\left(\sigma_{1}^{x}, \sigma_{1}^{y}, \sigma_{1}^{z}\right)$ and $\boldsymbol{\sigma}_{2}=\left(\sigma_{2}^{x}, \sigma_{2}^{y}, \sigma_{2}^{z}\right)$ with Pauli spin matrices $\sigma_{\alpha}^{i}, \alpha=1,2, i=x, y, z$, are of total length $s=1 / 2$. The first spin, $\boldsymbol{\sigma}_{1}$ is considered as the system and the second one, $\boldsymbol{\sigma}_{2}$ takes over the role of the environment. Every density matrix of the total system then assumes the form

$$
\rho=\frac{1}{4}\left(1+\mathbf{S}_{1} \cdot \boldsymbol{\sigma}_{1}+\mathbf{S}_{2} \cdot \boldsymbol{\sigma}_{2}+\boldsymbol{\sigma}_{1} \cdot \mathbf{C} \cdot \boldsymbol{\sigma}_{2}\right),
$$


where

$$
\mathbf{S}_{\alpha}=\left\langle\boldsymbol{\sigma}_{\alpha}\right\rangle
$$

denotes the Bloch vector of the spin $\alpha=1,2$ and the matrix

$$
\mathbf{C}=\left\langle\sigma_{1} \sigma_{2}\right\rangle
$$

denotes the correlation matrix of the two spins. The dot-product denotes the scalar product in three dimensions, e.g. $\mathbf{S}_{1} \cdot \boldsymbol{\sigma}_{1}=S_{1}^{x} \sigma_{1}^{x}+S_{1}^{y} \sigma_{1}^{y}+S_{1}^{z} \sigma_{1}^{z}$. The reduced density matrix of the system is given by the trace over the environment (i.e. the second spin), and hence becomes

$$
\rho_{S}=\operatorname{Tr}_{2} \rho=\frac{1}{2}\left(1+\mathbf{S}_{1} \cdot \boldsymbol{\sigma}_{1}\right) .
$$

Here we want to study the opposite direction, that is to go from $\rho_{S}$ to $\rho$. In particular, we look for conditions under which the respective blow-up map $R\left(\rho_{S}\right)=\rho$ is convex. We recall that the blow-up map is determined by the preparation process of the system. In the process of a preparation the state of the system is controlled by external fields $\mathbf{F}$ that ideally act only on the system, as given in eq. (1) for the equilibrium preparation. For the considered spin one can think of static magnetic fields. For interacting spins the two Bloch vectors and the correlation matrix will depend on the applied magnetic field.

We next investigate the requirement of the convexity of the blow-up map $R$. A necessary condition for this property to hold is the convexity of the domain of definition of $R$. This implies that for any pair of reduced density matrices $\rho_{S}^{\mathbf{F}_{k}}, k=1,2$ that result from two different values of the field, all convex linear combinations can be prepared by means of another value $\mathbf{F}_{3}$ of the field:

$$
\rho_{S}^{\mathbf{F}_{3}}=\lambda \rho_{S}^{\mathbf{F}_{1}}+(1-\lambda) \rho_{S}^{\mathbf{F}_{2}},
$$

with $\mathbf{F}_{3}$ being a uniquely defined function of the fields $\mathbf{F}_{1}, \mathbf{F}_{2}$ and $\lambda$. Using the general form of the reduced density matrix in (16) one finds a respective equation for the Bloch vectors, reading

$$
\mathbf{S}_{1}\left(\mathbf{F}_{3}\right)=\lambda \mathbf{S}_{1}\left(\mathbf{F}_{1}\right)+(1-\lambda) \mathbf{S}_{1}\left(\mathbf{F}_{2}\right) .
$$

This means that the Bloch vector of the system must be a uniquely invertible function of the external field. For the equilibrium preparation this is the case because then the derivatives of the Bloch vector components with respect to the field components coincide with the correlations of fluctuations of the first spin. These derivatives form the elements of the susceptibility matrix which is an invertible matrix in thermal equilibrium. Hence, $\mathbf{S}_{1}(\mathbf{F})$ has a uniquely defined inverse $\mathbf{F}\left(\mathbf{S}_{1}\right)$. We investigate the consequences of the convexity condition (10) of the blow-up map for the spin system. Using eqs. (17) and (13) for the total density matrix of the full system one finds analogous relations from eq. (10) for both the Bloch vector of the second spin and for the correlation matrix with the same field $\mathbf{F}_{3}$ that results from eq. (18), i.e.,

$$
\begin{aligned}
\mathbf{S}_{2}\left(\mathbf{F}_{3}\right) & =\lambda \mathbf{S}_{2}\left(\mathbf{F}_{1}\right)+(1-\lambda) \mathbf{S}_{2}\left(\mathbf{F}_{2}\right), \\
\mathbf{C}\left(\mathbf{F}_{3}\right) & =\lambda \mathbf{C}\left(\mathbf{F}_{1}\right)+(1-\lambda) \mathbf{C}\left(\mathbf{F}_{2}\right) .
\end{aligned}
$$

These are non-trivial conditions which in general will not be satisfied. Expressing next the fields $\mathbf{F}_{i}$ by the set of Bloch vectors $\mathbf{S}_{1}^{i}=\mathbf{S}_{1}\left(\mathbf{F}_{i}\right)$ that result for the respective fields we find by use of eq. (18) the following relations for the Bloch vectors of the second spin and the correlation matrix:

$$
\begin{aligned}
\mathbf{S}_{2}\left[\lambda \mathbf{S}_{1}^{1}+(1-\lambda) \mathbf{S}_{1}^{2}\right] & =\lambda \mathbf{S}_{2}\left[\mathbf{S}_{1}^{1}\right]+(1-\lambda) \mathbf{S}_{2}\left[\mathbf{S}_{1}^{2}\right], \\
\mathbf{C}\left[\lambda \mathbf{S}_{1}^{1}+(1-\lambda) \mathbf{S}_{1}^{2}\right] & =\lambda \mathbf{C}\left[\mathbf{S}_{1}^{1}\right]+(1-\lambda) \mathbf{C}\left[\mathbf{S}_{1}^{2}\right] .
\end{aligned}
$$

Hereby we introduced the notation $\mathbf{S}_{2}\left[\mathbf{S}_{1}\right]=\mathbf{S}_{2}(\mathbf{F})$ and $\mathbf{C}\left[\mathbf{S}_{1}\right]=\mathbf{C}(\mathbf{F})$. From these equations it follows that both $\mathbf{S}_{2}\left[\mathbf{S}_{1}\right]$ and $\mathbf{C}\left[\mathbf{S}_{1}\right]$ are affine functions, see Appendix $\mathrm{A}$ Therefore they can be represented as

$$
\begin{aligned}
\mathbf{S}_{2}\left[\mathbf{S}_{1}\right] & =\mathbf{A} \cdot \mathbf{S}_{1}+\mathbf{B}, \\
\mathbf{C}\left[\mathbf{S}_{1}\right] & =\mathbf{D} \cdot \mathbf{S}_{1}+\mathbf{E},
\end{aligned}
$$

where $\mathbf{B}$ is a constant vector, $\mathbf{A}$ and $\mathbf{E}$ are constant, second order tensors and $\mathbf{D}$ is a constant third order tensor. Hence, these quantities must neither depend on the applied field $\mathbf{F}$ nor on the Bloch vector $\mathbf{S}_{1}$. 


\section{AN EXPLICITE ILLUSTRATION}

We consider the equilibrium preparation for the following simple two-spin Hamiltonian as an example,

$$
H=-F_{z} \sigma_{1}^{z}+e \sigma_{2}^{z}+g \sigma_{1}^{x} \sigma_{2}^{x},
$$

Here we only allow for a field in the $z$-direction. The two spins interact by their $x$-components. We study the equilibrium preparation class at the fixed inverse temperature $\beta$ that results if the field $F_{z}$ assumes all possible values, $-\infty<F_{z}<\infty$ :

$$
\rho^{F_{z}}=Z^{-1} \exp \{-\beta H\}
$$

Because the Hamiltonian $H$ commutes with the operator $\sigma_{1}^{z} \sigma_{2}^{z}$ one can diagonalize $H$ in the eigenspaces of $\sigma_{1}^{z} \sigma_{2}^{z}$. This then yields the four eigenvalues $\mathcal{E}_{i}$

$$
\begin{array}{ll}
\mathcal{E}_{1}=-\sqrt{\left(F_{z}-e\right)^{2}+g^{2}}, & \mathcal{E}_{2}=\sqrt{\left(F_{z}-e\right)^{2}+g^{2}}, \\
\mathcal{E}_{3}=-\sqrt{\left(F_{z}+e\right)^{2}+g^{2}}, & \mathcal{E}_{4}=\sqrt{\left(F_{z}+e\right)^{2}+g^{2}},
\end{array}
$$

and the corresponding eigenprojection operators $P_{i}$ :

$$
\begin{array}{ll}
P_{i}=\frac{1}{4}\left(1+\sigma_{1}^{z} \sigma_{2}^{z}-\frac{F_{z}-e}{\mathcal{E}_{i}}\left(\sigma_{1}^{z}+\sigma_{2}^{z}\right)+\frac{g}{\mathcal{E}_{i}}\left(\sigma_{1}^{x} \sigma_{2}^{x}-\sigma_{1}^{y} \sigma_{2}^{y}\right)\right), & i=1,2, \\
P_{i}=\frac{1}{4}\left(1-\sigma_{1}^{z} \sigma_{2}^{z}-\frac{F_{z}+e}{\mathcal{E}_{i}}\left(\sigma_{1}^{z}-\sigma_{2}^{z}\right)+\frac{g}{\mathcal{E}_{i}}\left(\sigma_{1}^{x} \sigma_{2}^{x}+\sigma_{1}^{y} \sigma_{2}^{y}\right)\right), & i=3,4,
\end{array}
$$

such that $H=\sum_{i} \mathcal{E}_{i} P_{i}$ holds. The canonical density matrix $\rho^{F_{z}}$ at the inverse temperature $\beta$ is a mixture of the pure states $P_{i}$ with the Boltzmann weights $p_{i}=\exp \left\{-\beta \mathcal{E}_{i}\right\} /\left[2\left(\cosh \left(\beta \mathcal{E}_{1}\right)+\cosh \left(\beta \mathcal{E}_{2}\right)\right)\right]$, i.e.,

$$
\rho^{F_{z}}=\sum_{i=1}^{4} p_{i} P_{i}=\frac{1}{4}\left(1+S_{1 z} \sigma_{1}^{z}+S_{2 z} \sigma_{2}^{z}+C_{x x} \sigma_{1}^{x} \sigma_{2}^{x}+C_{y y} \sigma_{1}^{y} \sigma_{2}^{y}+C_{z z} \sigma_{1}^{z} \sigma_{2}^{z}\right) .
$$

The $x$ - and $y$-components of the two Bloch vectors vanish. The non-vanishing $z$-components read

$$
\begin{aligned}
& S_{1 z}=\beta\left(F_{z} \mathcal{F}_{+}\left(\beta \mathcal{E}_{1}, \beta \mathcal{E}_{3}\right)-e \mathcal{F}_{-}\left(\beta \mathcal{E}_{1}, \beta \mathcal{E}_{3}\right)\right), \\
& S_{2 z}=\beta\left(F_{z} \mathcal{F}_{-}\left(\beta \mathcal{E}_{1}, \beta \mathcal{E}_{3}\right)-e \mathcal{F}_{+}\left(\beta \mathcal{E}_{1}, \beta \mathcal{E}_{3}\right)\right),
\end{aligned}
$$

where the auxiliary functions $\mathcal{F}_{ \pm}(x, y)$ are defined by:

$$
\mathcal{F}_{ \pm}(x, y)=\frac{y \sinh (x) \pm x \sinh (y)}{x y(\cosh (x)+\cosh (y))} .
$$

Finally, the non-vanishing elements of the correlation matrix $\mathbf{C}$ are given by:

$$
\begin{aligned}
C_{x x} & =-\beta g \mathcal{F}_{+}\left(\beta \mathcal{E}_{1}, \beta \mathcal{E}_{3}\right) \\
C_{y y} & =\beta g \mathcal{F}_{-}\left(\beta \mathcal{E}_{1}, \beta \mathcal{E}_{3}\right) \\
C_{z z} & =\frac{\cosh \left(\beta \mathcal{E}_{1}\right)-\cosh \left(\beta \mathcal{E}_{3}\right)}{\cosh \left(\beta \mathcal{E}_{1}\right)+\cosh \left(\beta \mathcal{E}_{3}\right)} .
\end{aligned}
$$

As already stated above, in the present case of the equilibrium preparation the $z$-component of the Bloch vector of the first spin is a uniquely invertible function of the field magnetic $F_{z}$. This can be shown by inspection from eq. (29), see also Fig. 10 We note that the equilibrium preparation contains the pure system states $\rho_{S}=\frac{1}{2}\left(1 \pm \sigma_{z}\right)$ asymptotically in the infinite field limit $F_{z} \rightarrow \pm \infty$. If one also took into account fields that couple to the other spin components $\sigma_{x}, \sigma_{y}$, the eigenstates of these components could also be prepared asymptotically. In the case studied here, however, no other pure states than the eigenstates of $\sigma_{z}$ can be prepared. Thus, for this particular case one of the conditions of the Pechukas theorem to hold are not met. 


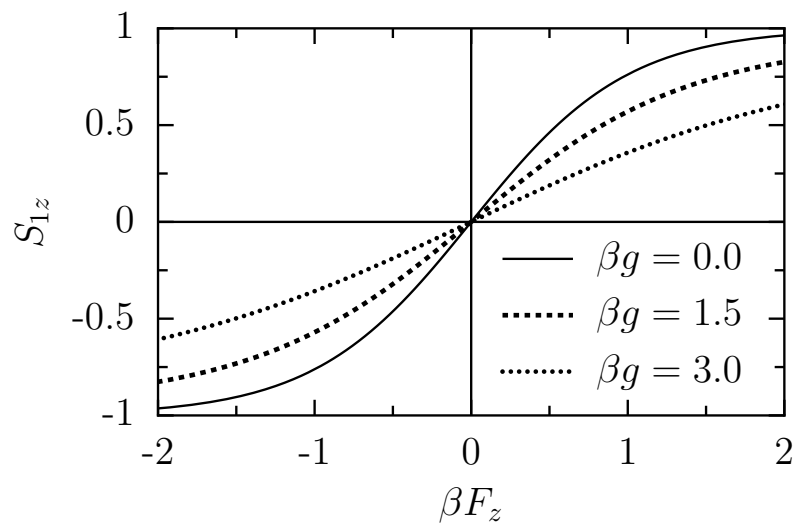

FIG. 1: The Bloch vector component $S_{z 1}$ as a function of the field $\beta F_{z}$ resulting from eq. (28) for $\beta e=1$. With increasing coupling parameter $\beta g=0.5,1,1.5$ the slope at $F_{z}=0$ decreases. Apparently, the functions $S_{z 1}(F)$ are monotonic and hence possess a unique inverse.

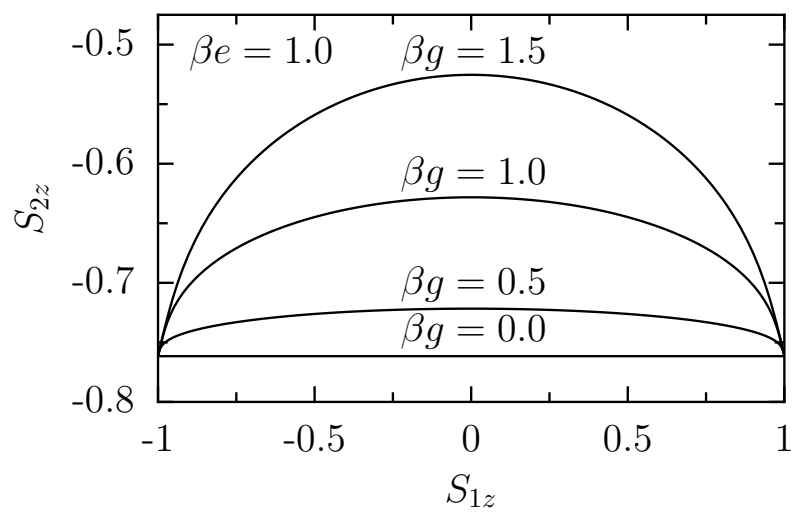

FIG. 2: The Bloch vector component $S_{z 2}$ as a function of $S_{z 1}$ resulting from eq. (28) for $\beta e=1$ and different values $\beta g=$ $0,0.5,1,1.5$. Note that a strictly linear dependence results only for the case of vanishing coupling $g=0$. An approximate linear regime exists in a neighborhood of $S_{1 z}=0$.

\section{A. Testing convexity of the blow-up map}

We now come to the discussion of the eqs. (21) which are necessary conditions for the blow-up map to be convex. Because in the considered preparation class only the $z$-component of the field is varied and because of the symmetries of the considered Hamiltonian these equations need only be checked as functions of $S_{z 1}$ for the Bloch vector component $S_{z 2}$ and the diagonal elements of the correlation matrix, respectively:

$$
\begin{aligned}
S_{z 2}\left[S_{z 1}\right] & =A S_{z 1}+B, \\
C_{x x}\left[S_{z 1}\right] & =D_{x x} S_{z 1}+E_{x x}, \\
C_{y y}\left[S_{z 1}\right] & =D_{y y} S_{z 1}+E_{y y}, \\
C_{z z}\left[S_{z 1}\right] & =D_{z z} S_{z 1}+E_{z z} .
\end{aligned}
$$

If the blow-up map were convex these equations would have to result from the eqs. (28) 30) by eliminating the external field $F_{z}$. There is no need to perform the lengthy calculation to see that the above equations hold only if the systemenvironment interaction is absent, i.e. if the coupling constant $g$ in the Hamiltonian vanishes. We note that with $\mathcal{E}_{1}\left(-F_{z}\right)=\mathcal{E}_{3}\left(F_{z}\right)$ and the symmetries of the auxiliary functions $\mathcal{F}_{ \pm}(x, y)= \pm \mathcal{F}_{ \pm}(y, x), S_{1 z}$ becomes an odd function whereas $C_{x x}$ is an even function of $F_{z}$. If eq. (31) was to hold, $D_{x x}$ would have to vanish and $C_{x x}$ would have to be a constant. This is true if and only if $g=0$. Figs. 2 and [ depict the dependences of $S_{z 2}$ and of the correlation functions, respectively, on $S_{z 1}$ for finite coupling strengths. In all cases, except for $g=0$, and for the correlation $C_{z z}$, the deviations from linearity are strikingly obvious. We note, however, that for small values of the Bloch vector component $S_{1 z}$ the component $S_{2 z}$ and correlation $C_{x x}$ are almost constant and the other correlations $C_{z z}$ and $C_{y y}$ 


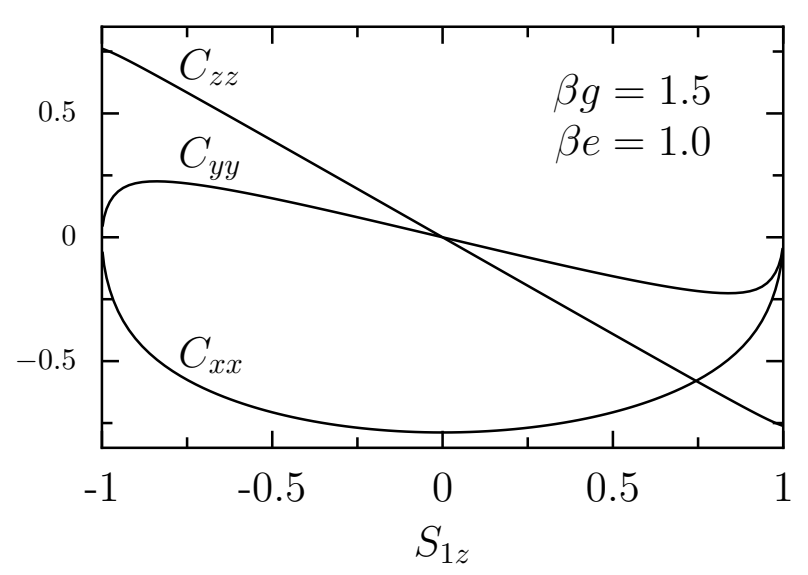

FIG. 3: The non-vanishing correlation functions $C_{x x}, C_{y y}, C_{z z}$ as a function of the Bloch vector component $S_{z}$ for $\beta e=1$ and $\beta g=1.5$. The correlation functions $C_{x x}$ and $C_{y y}$ clearly deviate from straight lines following from eq. (31). For the correlation functions also an approximately linear regime exists in a neighborhood of $S_{1 z}=0$.

are linear. This behavior is in accordance with a convex blow-up map. Below we will come back to the blow-up in the linear response regime when the external fields are small.

\section{DISCUSSION, IMPLICATIONS AND CONCLUSIONS}

We have illustrated Pechukas' verdict on the linear time evolution of open quantum systems [10] by a simple example. Moreover, we have demonstrated that the theorem holds for affine time evolutions: If two complete sets of pure system states can be prepared this more general class of evolution implies a factorizing preparation of the total initial density matrix where the environment density matrix must be independent of the system density matrix. Actually, Pechukas' original proof [10] applies as well in the affine case. Nowhere in the proof he made explicit use of the homogeneity condition, i.e. $R\left(\lambda \rho_{S}\right)=\lambda R\left(\rho_{S}\right), \lambda$ real, that would render an affine $R$ a linear map.

At first, this may only seem a modest generalization of the original conclusion but it sheds some light on the crucial role of the inhomogeneity term of (generalized) master equations which appears when the initial density matrix does not factorize. The present analysis excludes that this term is independent of the initial reduced density matrix and actually is not merely an inhomogeneity of the otherwise linear master equation but must depend on the reduced density matrix in a nonlinear way. In those cases, when a Markovian dynamics is approached for long times this nonlinear term must vanish for sufficiently large times. It will do so, however, in a characteristic manner that depends on the particular initial reduced density matrix.

In the present example only one set of pure system states, the eigenstates of $\sigma_{z}$, are preparable and yet the affinity of the blow-up map implies a factorizing preparation. We note that in general the condition on the number of preparable pure states of the Pechukas theorem cannot be relaxed. A counter example is provided by the work of Karrlein and Grabert [27] who considered a harmonic oscillator coupled to a bath of harmonic oscillators with a non-factorizing thermal preparation. This preparation allows for pure position states and still leads to a linear master equation.

Another example of a non-factorizing preparation that leads to a linear master equation results from the following preparation procedure: (i) start with a factorizing density matrix $\rho_{S 0} \rho_{B 0}$ at a time $t=-t_{0}, t_{0}>0$, (ii) turn on the interaction between the system and the bath, and (iii) use the density matrix that has evolved at $t=0$ as the result of the preparation. We term this preparation the factorize-and-wait preparation. The blow-up map $R\left(\rho_{S}\right)$ then assumes the form:

$$
R\left(\rho_{S}\right)=e^{-\frac{i}{\hbar} H t_{0}} G_{t_{0}}^{-1}\left(\rho_{S}\right) \rho_{B 0} e^{\frac{i}{\hbar} H t_{0}},
$$

where $G_{t}$ is the linear propagator of the reduced density matrix for the factorizing preparation, i.e.,

$$
\rho_{S}(t)=G_{t}\left(\rho_{S}(0)\right)=\operatorname{tr}_{B} e^{-\frac{i}{\hbar} H t_{0}} \rho_{S}(0) \rho_{B 0} e^{\frac{i}{\hbar} H t_{0}} .
$$

Here, the inverse of the propagator $G_{t}$ is needed in order to infer the proper system part of the factorizing density at $t=-t_{0}$ from the density matrix $\rho_{S}$ that is to be prepared at $t=0$. In view of possible fast relaxation processes and the build-up of system-bath correlations [23, 24, 26] after the interaction has been switched on, this inverse propagator 
will not be defined on the total set of possible density matrices [32]. Still, it is a linear operator and thus the blow-up map (32) also is linear. It is obvious, however, that no pure states of the system can be prepared at time $t=0$ in this way, i.e., that the factorize-and-wait preparation does not provide pure states. Therefore, the conditions for the Pechukas theorem are not met and the theorem is thus not in conflict with the linearity of the blow-up map of the entangled factorize-and-wait preparation.

Finally, we discuss the equilibrium preparation in the limit of weak external forces. In the region close to thermal equilibrium Mori's generalized quantum Langevin equations [33] provide a proper description of the time evolution of the set of system operators that couple to the external fields. In this case, the preparable density matrices of the total system follow from eq. (11) in linear approximation in the external fields $F_{j}$ and hence assume the linear response form [34]:

$$
\rho^{\mathbf{F}}=\rho^{\mathbf{0}}+\beta \sum_{i} \int_{0}^{1} d x\left(\rho^{\mathbf{0}}\right)^{1-x}\left(X_{i}-\left\langle X_{i}\right\rangle_{0}\right)\left(\rho^{\mathbf{0}}\right)^{x} F_{i}
$$

where $\rho^{\mathbf{0}}$ denotes the equilibrium density matrix of the total system in the absence of external fields and $\langle X\rangle_{0}$ the average of the operator $X$ with respect to the density matrix $\rho^{\mathbf{0}}$. The corresponding density matrix of the reduced system $\rho_{S}^{\mathbf{F}}=\operatorname{Tr}_{B} \rho^{\mathbf{F}}$ then becomes

$$
\rho_{S}^{\mathbf{F}}=\rho_{S}^{\mathbf{0}}+\beta \sum_{i} \int_{0}^{1} d x \operatorname{Tr}_{B}\left(\rho^{\mathbf{0}}\right)^{1-x}\left(X_{i}-\left\langle X_{i}\right\rangle_{0}\right)\left(\rho^{\mathbf{0}}\right)^{x} F_{i} .
$$

We recall that the external fields act on the system, i.e. that the conjugate operators $X_{i}$ are system operators. The expectation values of these operators with respect to the density matrix $\rho_{S}^{\mathbf{F}}$ are linear functions of the external fields by construction and can be written as:

$$
\left\langle X_{j}-\left\langle X_{j}\right\rangle_{0}\right\rangle \equiv \operatorname{Tr}_{S}\left(X_{j}-\left\langle X_{j}\right\rangle_{0}\right) \rho_{S}^{\mathbf{F}}=\sum_{i} \chi_{j i} F_{i}
$$

where the response matrix $\chi_{i j}$ is obtained by inserting eq. (34) into the middle term of eq. (36).

$$
\chi_{i j}=\beta \int_{0}^{1} d x \operatorname{Tr}\left(X_{i}-\left\langle X_{i}\right\rangle\right)\left(\rho^{\mathbf{0}}\right)^{1-x}\left(X_{j}-\left\langle X_{j}\right\rangle_{0}\right)\left(\rho^{\mathbf{0}}\right)^{x} .
$$

Because $\chi_{i j}$ is an invertible matrix we may solve eq. (36) for the external fields $F_{i}$

$$
F_{i}=\sum_{j} \chi^{-1}{ }_{i j}\left\langle X_{j}-\left\langle X_{j}\right\rangle_{0}\right\rangle
$$

In this way the external fields are expressed in terms of the linear functional $\left\langle X_{j}-\left\langle X_{j}\right\rangle_{0}\right\rangle$ of the system density matrix $\rho_{S}^{\mathbf{F}}$. Using this relation in eq. (34) we find for the blow-up map the affine form $R\left(\rho_{S}^{\mathbf{F}}\right)=L \rho_{S}^{\mathbf{F}}+\chi$ where

$$
\begin{aligned}
L \rho_{S}^{\mathbf{F}} & =\beta \sum_{i, j} \int_{0}^{1} d x\left(\rho^{\mathbf{0}}\right)^{1-x}\left(X_{i}-\left\langle X_{i}\right\rangle_{0}\right)\left(\rho^{\mathbf{0}}\right)^{x} \chi^{-1}{ }_{i j} \operatorname{Tr}_{S}\left(X_{j}-\left\langle X_{j}\right\rangle_{0}\right) \rho_{S}^{\mathbf{F}}, \\
\chi & =\rho^{\mathbf{0}} .
\end{aligned}
$$

With eq. (5) one obtains for the time evolution of the reduced density matrix

$$
\rho_{S}^{\mathbf{F}}(t)=\operatorname{Tr}_{B} U(t) L\left(\rho_{S}^{\mathbf{F}}\right) U^{\dagger}(t)+\operatorname{Tr}_{B} \rho^{\mathbf{0}}
$$

where we used that the total density matric $\rho^{\mathbf{0}}$ is invariant under the full time evolution. Actually this is a linear equation in the reduced density matrix $\rho_{S}$. In order to see this one puts $t=0$ in eq. (40), to express the trace of $\rho^{\mathbf{0}}$ over the environment in terms of the reduced density matrix $\rho_{S}$ : $\operatorname{Tr}_{B} \rho^{\mathbf{0}}=\rho_{S}-\operatorname{Tr}_{B} L\left(\rho_{S}\right)$. Hence, for the Mori preparation the time evolution of the reduced density matrix is linear. This is also in agreement with the findings for the above discussed model, see Figs. 2 and 3 


\section{APPENDIX A: CONVEX MAPS ARE AFFINE}

We prove that a differentiable convex map $M$ from a Banach space $B_{1}$ into a Banach space $B_{2}$ is also affine. The derivative of $M(x)$ at $x \in B_{1}$ is defined as the linear map $D M(x): B_{1} \rightarrow B_{2}$ that is tangential to $M$ at $x$. For further mathematical details see e.g. Ref. [35]:

$$
\lim _{\|h\|_{1} \rightarrow 0} \frac{\|M(x+h)-M(x)-D M(x) h\|_{2}}{\|h\|_{1}}=0,
$$

where $\|\cdot\|_{i}, i=1,2$ denotes the norm in the respective Banach space. The convexity of $M$ then requires that its domain of definition $\mathcal{D}(M)$ is convex, i.e. that with each pair $x, y$ of elements of $\mathcal{D}(M)$ also all convex linear combinations, $\lambda x+(1-\lambda) y$, with $0<\lambda<1$, belong to $\mathcal{D}(M)$. Moreover, this property is conserved under the convex $\operatorname{map} M$ :

$$
M(\lambda x+(1-\lambda) y)=\lambda M(x)+(1-\lambda) M(y) .
$$

Taking the derivative with respect to $\lambda$ one finds from (A2):

$$
D M(\lambda x+(1-\lambda) y)(x-y)=M(x)-M(y) .
$$

For $\lambda=0$ one obtains

$$
M(x)=D M(y) x+M(y)-D M(y) y .
$$

The first term on the right hand side is linear with respect to $x$ and the second and third term are independent of $x$ and hence $\mathrm{M}$ is affine.

\section{APPENDIX B: PECHUKA'S THEOREM}

Pechukas proved in Ref. [10] that a preparation is factorizing if the following conditions are satisfied: (i) the corresponding blow-up map of the preparation is convex; (ii) two different complete sets of pure system states can be prepared in the considered preparation class.

The proof consists of two steps. The first step is a consequence of the positivity of the blow-up map $R\left(\rho_{S}\right)$ and makes use of the possibility to prepare pure states of the system. Assume $\{|\psi\rangle\}$ is a pure state of the system that can be prepared. In the first step of the proof it is shown that the density matrix $\rho$ of the full system has the form

$$
\rho=R(|\psi\rangle\langle\psi|)=|\psi\rangle\langle\psi| \chi
$$

where the bath-density matrix $\chi$ in general depends on the system state $|\psi\rangle$.

For the proof we note that any density matrix of the total sytem can be represented as a weighted sum of products of pure system density matrices $\left|\psi_{i}\right\rangle\left\langle\psi_{i}\right|$ and bath density matrices $\chi_{i}$,

$$
\rho=\sum_{i} c_{i}\left|\psi_{i}\right\rangle\left\langle\psi_{i}\right| \chi_{i}
$$

where the states $\left|\psi_{i}\right\rangle$ are normalized and orthogonal on each other: $\left\langle\psi_{i} \mid \psi_{j}\right\rangle=\delta_{i, j}$, and the coefficients $c_{i}$ are not negative and add up to unity: $\sum_{i} c_{i}=1$. Taking the trace of $R(|\psi\rangle\langle\psi|)$ over the bath one recovers the pure state $|\psi\rangle\langle\psi|$ and on the other hand, using $\operatorname{Tr}_{B} \chi_{i}=1$ one finds from eq. (B22):

$$
|\psi\rangle\left\langle\psi\left|=\sum_{i} c_{i}\right| \psi_{i}\right\rangle\left\langle\psi_{i}\right| .
$$

Hence, all but one coefficients $c_{i}$ vanish and eq. (B1) holds.

Up to this point we have not made use of the convexity of the blow-up map and therefore the reduced bath density matrix $\chi$ may still depend on the system state $|\psi\rangle$.

In the second step of the proof we first take two pairs of orthonormal system states, $\left|\psi_{1}\right\rangle,\left|\psi_{2}\right\rangle$ and $\left|\varphi_{1}\right\rangle,\left|\varphi_{2}\right\rangle$ that span the same two-dimensional subspace:

$$
\left|\psi_{1}\right\rangle\left\langle\psi_{1}|+| \psi_{2}\right\rangle\left\langle\psi_{2}|=| \varphi_{1}\right\rangle\left\langle\varphi_{1}|+| \varphi_{2}\right\rangle\left\langle\varphi_{2}\right| \equiv P,
$$


where $P$ is the projection operator on this subspace. The absolute values of the mutual scalar products then are determined by an angle $\alpha$ :

$$
\begin{aligned}
\left|\left(\psi_{1}, \varphi_{1}\right)\right|^{2}=\cos ^{2} \alpha, \quad\left|\left(\psi_{1}, \varphi_{2}\right)\right|^{2}=\sin ^{2} \alpha, \\
\left|\left(\psi_{2}, \varphi_{1}\right)\right|^{2}=\sin ^{2} \alpha, \quad\left|\left(\psi_{2}, \varphi_{2}\right)\right|^{2}=\cos ^{2} \alpha .
\end{aligned}
$$

We assume that the two pairs of states $\psi_{i}$ and $\varphi_{i}$ can be prepared. According to the first step of the proof the full density matrix that corresponds to either of the pure states is a product of the pure state and a density matrix of the bath:

$$
R\left(\left|\psi_{i}\right\rangle\left\langle\psi_{i}\right|\right)=\left|\psi_{i}\right\rangle\left\langle\psi_{i}\left|\chi\left(\psi_{i}\right), \quad R\left(\left|\varphi_{i}\right\rangle\left\langle\varphi_{i}\right|\right)=\right| \varphi_{i}\right\rangle\left\langle\varphi_{i}\right| \chi\left(\varphi_{i}\right), \quad i=1,2,
$$

where $\chi\left(\psi_{i}\right)$ and $\chi\left(\varphi_{i}\right)$ are bath density matrices. We shall show that they all are the same. For this purpose we make use of the convexity of the blow up map and consider its action on the density matrix that is proportional to the projection $P$ onto the two dimensional subspace, $\rho_{S}=\frac{1}{2} P$. The action of the blow up map can be represented in either of two ways by convex combinations of pure states, see eq. (B44):

$$
\left|\psi_{1}\right\rangle\left\langle\psi_{1}\left|\chi\left(\psi_{1}\right)+\right| \psi_{2}\right\rangle\left\langle\psi_{2}\left|\chi\left(\psi_{2}\right)=\right| \varphi_{1}\right\rangle\left\langle\varphi_{1}\left|\chi\left(\varphi_{1}\right)+\right| \varphi_{2}\right\rangle\left\langle\varphi_{2}\right| \chi\left(\varphi_{2}\right) .
$$

Calculating the matrix elements with the four pure states $\psi_{i}, \varphi_{i}$, one obtains the following equations relating the reference density matrices $\chi\left(\psi_{i}\right)$ and $\chi\left(\varphi_{i}\right)$ :

$$
\left(\begin{array}{c}
\chi\left(\psi_{1}\right) \\
\chi\left(\psi_{2}\right)
\end{array}\right)=\left(\begin{array}{cc}
|\cos \alpha|^{2} & |\sin \alpha|^{2} \\
|\sin \alpha|^{2} & |\cos \alpha|^{2}
\end{array}\right)\left(\begin{array}{c}
\chi\left(\varphi_{1}\right) \\
\chi\left(\varphi_{2}\right)
\end{array}\right)
$$

and

$$
\left(\begin{array}{c}
\chi\left(\varphi_{1}\right) \\
\chi\left(\varphi_{2}\right)
\end{array}\right)=\left(\begin{array}{cc}
|\cos \alpha|^{2} & |\sin \alpha|^{2} \\
|\sin \alpha|^{2} & |\cos \alpha|^{2}
\end{array}\right)\left(\begin{array}{c}
\chi\left(\psi_{1}\right) \\
\chi\left(\psi_{2}\right)
\end{array}\right)
$$

We may exclude the trivial cases when the pairs $\psi_{i}$ and $\varphi_{i}$ coincide and either $\cos ^{2} \alpha=1$ or $\sin ^{2} \alpha=1$. In all other cases, the four equations only have a solution with $\chi\left(\psi_{1}\right)=\chi\left(\psi_{2}\right)=\chi\left(\varphi_{1}\right)=\chi\left(\varphi_{2}\right)$. Hence, the bath density matrix is identical for all preparable system density matrices in the considered subspace such that a factorizable state of the total system results on this subspace.

In order to apply this argument to higher dimensional system Hilbert spaces it must be possible to prepare sufficiently many pure system states. Starting as above with a two dimensional subspace that can be spanned by two pairs of preparable bases $\left\{\psi_{i}\right\},\left\{\varphi_{i}\right\}, i=1,2$ one next considers the subspace spanned by either $\left|\psi_{1}\right\rangle$ and $\left|\psi_{3}\right\rangle$ or by $\left|\varphi_{1}\right\rangle$ and $\left|\varphi_{3}\right\rangle$ and finds $\chi\left(\psi_{1}\right)=\chi\left(\psi_{3}\right)$ for the reference bath operators in the density matrix of the full system. That means that two different sets of pure states spanning the system's Hilbert space must be preparable.

\section{ACKNOWLEDGMENTS}

The authors thank Gert-Ludwig Ingold and Juan Parrondo for valuable discussions and hints. This work was supported by the Research network "Quanteninformation entlang der A8" and by the Sonderforschungsbereich 631 of the Deutsche Forschungsgemeinschaft.

[1] U. Weiss, Quantum Dissipative systems, World Scientific, Singapore, 1999.

[2] W. Pauli, Probleme der modernen Physik, ed. P. Debye, Hirzel Verlag, Leipzig, 1928, p. 30.

[3] L. van Hove, Physica 23, 441 (1957).

[4] F. Haake, in Springer Tracts in Modern Physics, Vol. 66, Springer, Berlin 1974, p. 98.

[5] K. Blum, Density Matrix Theory and Applications, 2-nd edition, New York: Plenum, 1996.

[6] V. May, O. Kühn, Charge and Energy Transfer Dynamics in Molecular Systems, Wiley-VCH Verlag, Berlin, 2003.

[7] See for example the special issue of the Scientific American, 285, No. 3, Sept. 2001.

[8] M. A. Nielsen, I. L. Chuang, Quantum Computation and Quantum Information, Cambridge University Press, Cambridge, 2000.

[9] E. C. G. Sudarshan, P. M. Mathews, J. Rau, Phys. Rev. 121, 920 (1961).

[10] P. Pechukas, Phys. Rev. Lett. 73, 1060 (1994). 
[11] P. Pechukas, Phys. Rev. Lett. 75, 3021 (1995).

[12] V. Gorini, A. Kossakowski, E. C. G. Sudarshan, J. Math. Phys. 17, 821 (1976).

[13] G. Lindblad, Rep. Math. Phys. 10, 393 (1976).

[14] H. Grabert, U. Weiss, P. Talkner, Z. Phys. B 55, 87 (1984).

[15] P. S. Riseborough, P. Hänggi, U. Weiss, Phys. Rev. A 31, 471 (1985).

[16] P. Talkner, Ann. Phys. 167, 390 (1986).

[17] K. M. Fonseca Romero, M. C. Nemes, Phys. Lett. A 235, 432 (1997).

[18] K. M. Fonseca Romero, M. C. Nemes, Physica A 325333 (2003).

[19] H. Grabert, P. Talkner, P. Hänggi, Z. Phys. B 26, 389 (1977).

[20] H. Grabert, P. Talkner, P. Hänggi, H. Thomas, Z. Phys. B 29, 273 (1978).

[21] H. Grabert, P. Hänggi, P. Talkner, J. Stat. Phys. 22, 537 (1980).

[22] H. Grabert, Schramm, G.-L. Ingold, Phys. Rep. 168, 115 (1988).

[23] W. Bez, Z. Phys. B 39, 319 (1980).

[24] A. Suarez, R. Silbey, I. Oppenheim, J. Chem. Phys. 97, 5101 (1992).

[25] J. S. Canizares, F. Sols, Physica A 212, 181 (1994).

[26] S. Gnutzmann, F. Haake, Z. Phys. B 101, 263 (1996).

[27] R. Karrlein, H. Grabert, Phys. Rev. 55, 153 (1997).

[28] U. Zürcher, P. Talkner, Phys. Rev. A 42, 3267 (1990).

[29] S. Kohler, T. Dittrich, P. Hänggi, Phys. Rev E 55, 300 (1997).

[30] G.-L. Ingold Path Integrals and Their Application to Dissipative Quantum Systems in: Coherent Evolution in Noisy Environments, ed. by A. Buchleitner and K. Hornberger, Lecture Notes in Physics, vol. 611, pp. 1-53, Springer, 2002.

[31] H. Spohn, Rev. Mod. Phys. 52, 569 (1980).

[32] P. Hänggi, H. Thomas, H. Grabert, P. Talkner, J. Stat. Phys. 18, 155 (1978).

[33] H. Mori, Prog. Theor. Phys. 33, 423 (1965).

[34] R. Kubo, Rep. Prog. Phys. 29, 255 (1966).

[35] R. Abraham, J. E. Marsden, T. Ratiu, Manifolds, Tensor Analysis, and Applications, Addison Wesley Pub., London, 1983. 